\title{
Reply to Letter to the Editor "Staging of acute leukemia based on central nervous system involvement alone: Is it appropriate?"
}

\section{Sumit Gupta, A. Lindsay Frazier and Joanne F. Aitken}

To the Editor: On behalf of our fellow authors, we thank Drs Totadri and Bansal for their

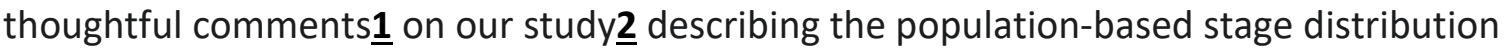
among Australian children with hematologic malignancies. Their main point focuses not on our study specifically but rather the appropriateness of staging acute leukemia using central nervous system (CNS) involvement, as recommended by the Toronto Paediatric Cancer Stage Guidelines. $\underline{\mathbf{3}}$ They have two main arguments: that there are stronger prognosticators in acute leukemia and that CNS involvement is rare.1

We agree that there are many important prognosticators in acute leukemia in addition to CNS involvement. However, as Drs Totadri and Bansal themselves state, $\underline{\mathbf{1}}$ stage is defined as the anatomic extent of disease. Thus, factors such as age, immunophenotype, leukemia genetics, and treatment response are all important prognosticators that nonetheless cannot be considered part of any staging system. Whether population-based registries should collect important nonstage prognosticators is a question of increasing importance that will be discussed in an upcoming meeting of cancer registrars, epidemiologists, and clinicians. Indeed, this is relevant for not just acute leukemia but many other malignancies. One could plausibly argue that $\mathrm{N}$-myc status in neuroblastoma, or molecular subtype in medulloblastoma, play as important of a prognostic role as stage.

Drs Totadri and Bansal $\underline{\mathbf{1}}$ also point out that in a large Children's Oncology Group study of children with acute lymphoblastic leukemia (ALL), only $1.5 \%$ had CNS3 status. In the same 
study however, another $10.2 \%$ of children had CNS2 status. The 5-year event-free survival associated with both CNS2 and CNS3 status was identical at 76\%, as compared to $85 \%$ among children with CNS negativity. $\underline{4}$ Both CNS2 and CNS3 status are recommended to be collected by the Toronto Guidelines, whether separately or collapsed into overall CNS positivity.

We again thank Drs Totadri and Bansal for the discussion they have initiated. Collecting the most relevant childhood cancer data on a population-based level will help inform researchers, clinicians, and health policymakers, and ultimately lead to improvements in cancer outcomes for this population.

\section{REFERENCES}

Totadri S, Bansal D. Staging of acute leukemia based on central nervous system involvement alone: Is it appropriate? Paediatr Blood Cancer. 2019. In press.

Youlden DR, Gupta S, Frazier AL, et al. Stage at diagnosis for children with blood cancers in Australia: application of the Toronto Paediatric Cancer Stage Guidelines in a populationbased national childhood cancer registry. Pediatr Blood Cancer. 2017; 64:e26683.

Gupta S, Aitken JF, Bartels U, et al. Paediatric cancer stage in population-based cancer registries: the Toronto consensus principles and guidelines. Lancet Oncol. 2016; 17: e163e172.

Winick NJ, Devidas M, Maloney K, et al. The impact of initial cerebrospinal fluid (CSF) findings on outcome among patients with $\mathrm{NCl}$ standard (SR) and high-risk (HR) B- 
lymphoblastic leukemia (ALL): a report from the Children's Oncology Group (COG) Studies AALL0331 and AALL0232. J Clin Oncol. 2014; 23:Abstr 10016. 